\title{
Coupled-Mode Envelope Solitary Waves in a Pair of Cubic Schrödinger Equations with Cross Modulation: Analytical Solution and Collisions with Application to Rossby Waves
}

\author{
Benkui Tan \\ Dept. of Geophysics, Peking University, Beijing 100871, People's Republic of \\ China, (dqgchw@ibmstone.pku.edu.cn). \\ John P. Boyd \\ Dept. of Atmospheric, Oceanic and Space Science and Program in Scientific \\ Computing, 2455 Hayward Avenue, Ann Arbor MI 48109, \\ (jpboyd@engin.umich.edu; http://www-personal.engin.umich.edu:/ \\ jpboyd/; Phone: (313) 764-3338; FAX: (313) 764-5137).
}

Keywords: envelope solitary wave, coupled-mode soliton, soliton collisions

\begin{abstract}
Coupled Nonlinear Schrödinger equations, linked by cross modulation terms, arise in both nonlinear optics and in Rossby waves in the atmosphere and ocean. In this paper, we derive exact, analytic solutions for the "bright" coupled-mode soliton, for which the envelope in each mode asymptotes to zero at spatial infinity, and for its spatially periodic generalization. We then numerically study the collisions of the coupled-mode solitary wave both with a conventional envelope soliton, confined to a single mode, and also with a second coupled-mode solitary wave. The collisions are sensitive to both the relative speed and phase of the solitons. In some parameter ranges, the collisions are nearly elastic, but in others, one or both solitons fission.
\end{abstract}

$\overline{1}$ This work was supported by the National Natural Science Foundation of China through grant 49375239 and by the National Science Foundation through grant OCE9521133.

Preprint submitted to Elsevier Preprint 24 September 2000 


\section{Introduction}

The Nonlinear Schrödinger (NLS) equation has been derived as a model for weakly nonlinear wave packets in a wide variety of physical systems by means of the perturbative algorithm known as the method of multiple scales. When linear wave theory allows for more than one mode, then one may derive independent Nonlinear Schrödinger equations for wave packets in each mode. In the small amplitude expansion, different modes do not interact to lowest order. However, when there are resonances, that is, when the linear phase speeds are approximately equal for waves in different modes, then the nonlinear interaction between the resonant modes is amplified. Two independent NLS equations must then be replaced by a coupled pair of equations which have the form

$$
\begin{aligned}
& i \frac{\partial A_{1}}{\partial T}+\alpha_{1} \frac{\partial^{2} A_{1}}{\partial X^{2}}+\left(\sigma_{1}\left|A_{1}\right|^{2}+\nu_{12}\left|A_{2}\right|^{2}\right) A_{1}=0 \\
& i \frac{\partial A_{2}}{\partial T}+\alpha_{2} \frac{\partial^{2} A_{2}}{\partial X^{2}}+\left(\sigma_{2}\left|A_{2}\right|^{2}+\nu_{21}\left|A_{1}\right|^{2}\right) A_{2}=0
\end{aligned}
$$

where $A_{1}$ and $A_{2}$ are the complex amplitudes or "envelopes" of the two wave packets, respectively. We shall dub this the "Coupled-Nonlinear Schrödinger (CNLS)" system. In the language of the method of multiple scales, $T$ is the slow time and $X$ is the slow space variable moving at the linear group velocity. The coefficients $\alpha_{1}$ and $\alpha_{2}$ are the so-called "dispersion coefficients", $\sigma_{1}$ and $\sigma_{2}$ are the "Landau constants" describing the self-modulation of the wave packets, and $\nu_{12}$ and $\nu_{21}$ are the "coupling constants" of the cross-modulation between the two wave packets. The complete wave disturbance is the product of each envelope with the appropriate sinusoidal "carrier wave" and a mode factor that describes the dependence of the packet on the spatial coordinates $y$ and $z$; the packet propagates only in the $x$-direction. The dispersion, Landau and coupling constants are functions of the wavenumber of the carrier wave, the modal structure, and parameters of the wave medium.

Tan and Liu [14] have derived this system for marginally unstable baroclinic waves in a two-layer atmospheric model. The different "modes" of the CNLS system are the two vertical eigenfunctions of the two-layer model. Tan[13] derived this same system for Rossby waves in a barotropic model of the atmosphere. In this case, the interacting wave packets are in different latitudinal modes. In this work, we shall not discuss the derivation of Eq. 15 further, but instead restrict ourselves to understanding the dynamics of the CNLS system itself. However, the numerical values of the dispersion, Landau and coupling constants will be taken from those two papers of the senior author's.

The coupled-NLS has also been extensively studied in nonlinear optics $[9,22,15,10,16,11,8]$ 
$[1,7,21,17-20, ?, ?]$. However, for birefringent fibers, there are connections between the dispersion, nonlinear and coupling constants. After the number of coefficients has been reduced by rescaling the space and time coordinates and the amplitudes, only a single parameter remains. In contrast, in the geophysical applications that motivated our work, there are no simple relation between the coefficients, and even after rescaling, three coefficients different from one remain. It follows that while we can borrow many ideas from this large body of previous work on the coupled-NLS, the extra parameters in the geophysical applications require us to also pursue previously unexplored realms of parameter space.

In the next section, we describe the scaling of the coupled-NLS (CNLS) system. We can significantly reduce the number of parameters, but are still left with too many to completely explore the parameter space. We also give the coupled system of two ODEs, which we shall dub the Steadily-oscillating CoupledNonlinear Schrödinger (SCNLS) system, and its scaling. This, alas, has three parameters left after changing the unknowns and coordinates: a nondimensional frequency ratio plus two rescaled cross-modulation coefficients.

Sec. 3 reviews the single-mode solitary waves (those of the ordinary NLS equation) and three classes of coupled-mode solitons. All have been known before, but we generalize these to more parameters and, for the most important family, also extend it from the soliton to spatially periodic elliptic functions.

In the next two sections, we describe collisions of coupled-mode solitons with either solitary waves residing in a single mode only or with other coupled-mode solitary waves.

\section{Scaling Analysis and Galilean Invariance}

Theorem 1 (Rescaling: Time-Dependent System) By rescaling the spatial coordinate and both amplitudes, it is possible to reduce the number of nontrivial coefficients of the coupled-NLS system from six to three. The simplified system is

$$
\begin{array}{r}
i A_{T}+A_{\zeta \zeta}+|A|^{2} A+\tilde{\nu}_{12}|B|^{2} A=0 \\
i B_{T}+\alpha B_{\zeta \zeta}+|B|^{2} B+\tilde{\nu}_{21}|A|^{2} B=0
\end{array}
$$

where the subscripts denote differentiation with respect to the subscripted variables and where the new unknowns and coefficients are

$$
A \equiv \sqrt{\sigma_{1}} A_{1}, \quad B \equiv \sqrt{\sigma_{2}} A_{2}
$$




$$
\begin{gathered}
\zeta \equiv \sqrt{1 / \alpha_{1}} X \\
\tilde{\nu}_{12}=\frac{\nu_{12}}{\sigma_{2}}, \quad \tilde{\nu}_{21}=\frac{\nu_{21}}{\sigma_{1}}, \quad \alpha=\frac{\alpha_{2}}{\alpha_{1}}
\end{gathered}
$$

Proof: Change of unknowns and coordinates in the original, unscaled equation.

The steadily-oscillating solutions of this are solitons and their spatially periodical generalizations, which we shall call "cnoidal" waves even when not described by elliptic functions. Solitons and cnoidal waves are obtained by assuming

$$
A_{1}=\exp \left(i \mu^{2} \alpha_{1} t\right) u(X), \quad A_{2}=\exp \left(i \beta^{2} \alpha_{2} t\right) v(X)
$$

where $\mu, \beta$ and $u, v$ are assumed to be real. Note that in spite of the crossmodulation, the frequencies in the two modes are generally different. Substitution gives a set of two coupled ODEs:

$$
\begin{aligned}
& u_{X X}-\mu^{2} u+\frac{\sigma_{1}}{\alpha_{1}} u^{3}+\frac{\nu_{12}}{\alpha_{1}} u v^{2}=0 \\
& \text { SCNLS } \\
& v_{X X}-\beta^{2} v+\frac{\sigma_{2}}{\alpha_{2}} v^{3}+\frac{\nu_{21}}{\alpha_{2}} u^{2} v=0
\end{aligned}
$$

Theorem 2 (Rescaling: ODE System) By rescaling the spatial coordinate and both amplitudes, it is possible to reduce the number of non-trivial coefficients of the Steadily-oscillating Coupled-NLS system from six to three. The simplified system is

$$
\begin{array}{r}
U_{\zeta \zeta}-U+2 U^{3}+\tilde{\nu}_{12} V^{2} U=0 \\
V_{\zeta \zeta}-\epsilon^{2} V+2 V^{3}+\tilde{\nu}_{21} U^{2} V=0
\end{array}
$$

where the new unknowns and coefficients are

$$
\begin{gathered}
U \equiv \frac{1}{\mu} \sqrt{\frac{\sigma_{1}}{2 \alpha_{1}}} u, \quad V \equiv \frac{1}{\mu} \sqrt{\frac{\sigma_{2}}{2 \alpha_{2}}} v \\
\epsilon \equiv \frac{\beta}{\mu} \\
\tilde{\nu}_{12} \equiv \frac{2 \alpha_{2} \nu_{12}}{\alpha_{1} \sigma_{2}}, \quad \tilde{\nu}_{21} \equiv \frac{2 \alpha_{1} \nu_{21}}{\alpha_{2} \sigma_{1}} \\
\zeta=\mu X
\end{gathered}
$$


Proof: Change of unknowns and coordinates in the original, unscaled equation.

To compute the solitary and cnoidal waves, it is always sufficient to solve this system of two ODEs. However, to collide solitary waves, it is necessary to shift the steadily-oscillating solitary waves into a frame of reference such that they move at some translational velocity $V$. The following theorem, which applies directly to all solutions, solitary or not, of the time-dependent CNLS equations, allows us to do this.

Theorem 3 (Galilean Invariance) If $A_{1}(X, T), A_{2}(X, T)$ are a solution to the Coupled-NLS system

$$
\begin{aligned}
& i \frac{\partial A_{1}}{\partial T}+\alpha_{1} \frac{\partial^{2} A_{1}}{\partial X^{2}}+\left(\sigma_{1}\left|A_{1}\right|^{2}+\nu_{12}\left|A_{2}\right|^{2}\right) A_{1}=0 \\
& i \frac{\partial A_{2}}{\partial T}+\alpha_{2} \frac{\partial^{2} A_{2}}{\partial X^{2}}+\left(\sigma_{2}\left|A_{2}\right|^{2}+\nu_{21}\left|A_{1}\right|^{2}\right) A_{2}=0
\end{aligned}
$$

then another solution is

$$
\begin{aligned}
& \tilde{A}_{1} \equiv A_{1}(X-V T, T) \exp \left(i \xi_{1} X\right) \exp \left(-i \alpha_{1} \xi_{1}^{2} T\right) \\
& \tilde{A}_{2} \equiv A_{2}(X-V T, T) \exp \left(i \xi_{2} X\right) \exp \left(-i \alpha_{2} \xi_{2}^{2} T\right)
\end{aligned}
$$

where the velocity $V$ is

$$
V=2 \alpha_{1} \xi_{1}=2 \alpha_{2} \xi_{2} \leftrightarrow \xi_{2}=\xi_{1} \frac{\alpha_{1}}{\alpha_{2}}
$$

which implies that the constants $\xi_{1}$ and $\xi_{2}$ must be proportional as indicated.

Proof. Substitution into the CNLS system.

\section{Solitary and Cnoidal Waves}

The system of two ordinary differential equations in two real-valued unknowns which describes solitary and cnoidal waves, Eqs.(8), has a wide variety of solutions. Most can be computed only by numerical methods, but a number of special cases can be described by explicit, analytical exact solutions or approximations. These analytical special cases are important because they are initial points for computing solution branches through numerical continuation. 


\subsection{Single-Mode Solitons}

If either $u \equiv 0$ or $v \equiv 0$, then the cross-modulation is zero and the ODE for the non-zero component is identical to the steadily-oscillation form of the usual Nonlinear Schrödinger (NLS) equation. It is solved by the usual NLS soliton:

$$
u=\sqrt{\frac{2 \alpha_{1}}{\sigma_{1}}} \mu \operatorname{sech}(\mu x), \quad v \equiv 0
$$

or for a soliton in the $v$-mode:

$$
u \equiv 0, \quad v=\sqrt{\frac{2 \alpha_{2}}{\sigma_{2}}} \mu \operatorname{sech}(\beta x)
$$

We shall refer to either of these cases as a "single-mode" soliton. We shall label the three classes of solitary waves in the next three sections as "coupledmode" solitons because they have non-zero amplitude in both $A_{1}$ and $A_{2}$. In the nonlinear optics literature, these are sometimes called "symbiotic" solitary waves or more often "vector" solitons.

\subsection{Equal-Frequency Solitary and Cnoidal Waves}

Set $\beta=\mu$ and define

$$
u=a w\left(x ; \mu^{2}\right), \quad v=b w\left(x ; \mu^{2}\right)
$$

where $w(x ; c)$ is a solution to

$$
w_{x x}-\mu^{2} w+2 w^{3}=0
$$

Substituting into the ODE system and collecting terms in $w^{3}$ and imposing the condition that the coefficients must equal 2 gives the $2 \times 2$ matrix system:

$$
\left|\begin{array}{cc}
\frac{\sigma_{1}}{\alpha_{1}} & \frac{\nu_{12}}{\alpha_{1}} \\
\frac{\nu_{21}}{\alpha_{2}} & \frac{\sigma_{2}}{\alpha_{2}}
\end{array}\right|\left|\begin{array}{l}
a^{2} \\
b^{2}
\end{array}\right|=\left|\begin{array}{l}
2 \\
2
\end{array}\right|
$$

The solution is

$$
\begin{aligned}
& a^{2}=2 \frac{\alpha_{1} \sigma_{2}-\alpha_{2} \nu_{12}}{\sigma_{1} \sigma_{2}-\nu_{12} \nu_{21}} \\
& b^{2}=2 \frac{\alpha_{2} \sigma_{1}-\alpha_{1} \nu_{21}}{\sigma_{1} \sigma_{2}-\nu_{12} \nu_{21}}
\end{aligned}
$$


Solutions to the equation for $w$ are given in Appendix D, Sec. D.2.2 on pgs. 498499 of [4] and in many standard texts on elliptic functions. Both cnoidal and dnoidal periodic solutions exist. The limiting solitary waves for both branches are

$$
w= \pm \mu \operatorname{sech}(\mu x)
$$

\subsection{Father-Daughter Approximations}

The key assumptions are (i) $\max |v|<<\max |u|$ and (ii) $u$ is a single mode solitary wave, i. e.,

$$
u=\sqrt{\frac{2 \alpha_{1}}{\sigma_{1}}} \mu \operatorname{sech}(\mu x)
$$

Because of the assumed smallness of $v$, the self-interaction of $v$ can be neglected, which simplifies the second ODE to

$$
v_{x x}-\beta^{2} v+\left\{\frac{\nu_{21}}{\alpha_{2}} \frac{2 \alpha_{1}}{\sigma_{1}} \mu^{2}\right\} \operatorname{sech}^{2}(\mu x) v=0
$$

The change of coordinate

$$
z \equiv \tanh (\mu x)
$$

the introduction of the frequency ratio $\epsilon \equiv \beta / \mu$ (as in Eq.12) and a parameter $\rho$ defined by

$$
\rho(\rho+1) \equiv\left\{\frac{\nu_{21}}{\alpha_{2}} \frac{2 \alpha_{1}}{\sigma_{1}}\right\}
$$

transforms Eq. (27) into the equation solved by the Associated Legendre functions:

$$
\left\{\left(1-z^{2}\right) v_{z z}-2 z v_{z}\right\}-\frac{\epsilon^{2}}{1-z^{2}} v+\rho(\rho+1) v=0
$$

The eigenmodes of this homogeneous linear equation are

$$
P_{\rho}^{\epsilon}(z), \quad j=0,1,2, \ldots, j_{\max }
$$

where the frequency ratio $\epsilon$ is forced to satisfy the following:

$$
\epsilon=\rho-j, \quad j=0,1,2, \ldots, j_{\max }
$$


where the mode number $j$ is always a non-negative integer and where $j_{\max }$ is the largest integer such that $\epsilon>0$.

Yang(1997, pg. 96) gives the lowest three modes:

$$
\begin{gathered}
P_{\rho}^{\rho}(z) \equiv\left(1-z^{2}\right)^{\rho / 2}=\operatorname{sech}^{\rho}(\mu x) \\
P_{\rho}^{\rho-1}(z)=\left(1-z^{2}\right)^{\rho / 2} \frac{z}{\left(1-z^{2}\right)^{1 / 2}}=\operatorname{sech}^{\rho}(\mu x) \sinh (\mu x) \\
P_{\rho}^{\rho-2}(z)=\operatorname{sech}^{\rho}(\mu x)\left\{1+2(1-\rho) \sinh ^{2}(\mu x)\right\}
\end{gathered}
$$

However, only the lowest mode exists if $\rho<1$.

As the frequency ratio $\epsilon$ is varied from one of the eigenvalues specified by Eq.(32), $v$ grows to finite amplitude and gradually changes its shape. Yang [19] has computed the first order corrections to these Legendre modes, but numerical methods are needed to accurately trace the full solution branch emerging from these "father-daughter" solutions. The $u$ soliton is the "father"; the infinitesimal amplitude $v$, solving the Associated Legendre equation, is the "daughter".

\subsection{Multiple-Scale Compositions}

When $\beta<<\mu$, there is another branch of solitons in which $u$ is very narrow (with width $O(1 / \mu) \sim O(1)$ ) whereas $v$ consists of two sech-shaped peaks which are very broad $O(1 / \beta)$. Without cross-modulation, the ODE for $v$ reduces to the ordinary NLS equation, which does not have any two-humped solitary waves. The narrow spike in $u$ acts as a "glue" to modify $v$ between the two peaks so that a solitary wave with two peaks is possible. Akhmediev and Ankiewicz [2] and Haelterman and Sheppard [7] independently discovered and numerically computed this branch of solutions.

Although both groups of authors were pessimistic about analytical approximations, we are presently applying the method of matched asymptotic expansions to these solutions. Our analysis will be published in another place.

\subsection{Solution Branches}

The analytically-treatable coupled-mode solitons are but special cases. Each is the limit of a branch of soliton solutions. The rest of the branch can be computed numerically through the continuation method as explained in Sec. 5 
below. The key idea is to vary a parameter in small steps using previouslycomputed solutions to initialize the Newton's iteration for the current parameter value. The analytical special cases are important because they are the initial points on each solution branch.

Fig. 1 illustrates a solution branch emerging from the equal-frequency soliton for the same coefficients of the CNLS system as illustrated in Fig. 3 below. The branch terminates at $\beta=0.33263$ where max $|v|$ goes to zero and $v$ becomes the Associated Legendre function, well-described by the "father-daughter" approximation explained above. As $\beta$ is decreased from its initial value of one to $\beta=1 / 3$, the $u$ component changes very little so that all the curves for different $\beta$ are almost superimposed on top of one another. This happens because the width of $u$ is controlled primarily by the frequency parameter $\mu$, which is fixed at one. The other component $v$ becomes smaller and smaller and wider and wider as $\beta$ decreases. Although we stopped the numerical calculation at $\beta=1 / 3$, just short of the bifurcation point, Fig. 2 shows that the Legendre function, which for the lowest Legendre mode is just $\operatorname{sech}(\mu X)$ raised to the $n$-th power where $n=0.33263$ for this set of CNLS coefficients, is a very good approximation to $v$ for $\beta=1 / 3$.

In the rest of this article, we shall restrict attention to the equal-frequency coupled-mode solitons. Stability analyses [18-20] suggest that the other species of solitary waves are easily disassociated. The equal-frequency solitary waves are merely one point on a branch of solutions, but it is when the solitary waves have equal width and frequency that they are likely to have the strongest and most varied interactions. However, we have reviewed several types of solitons to emphasize that the parameter space is so large, and the solitary waves so varied, that in a single article we can only hope to offer an interesting sample rather than an exhaustive discussion of soliton-soliton interactions for the CNLS system.

\section{Stability and Cross-Modulation}

The Nonlinear Schrödinger equation was so named because it is identical in form to the Schrödinger equation of quantum mechanics with the nonlinear terms playing the role of the potential energy. For the ordinary uncoupled NLS, solitons are possible only when the nonlinear coefficient $\sigma$ has the same sign as the dispersion coefficient $\alpha$ because it is only then that the nonlinear self-interaction creates an attractive force or what in mechanics is called a "potential well" [14,13].

The cross-modulation terms are the glue that holds coupled-mode solitary waves together. It follows that such solitons are likely to be unstable unless 

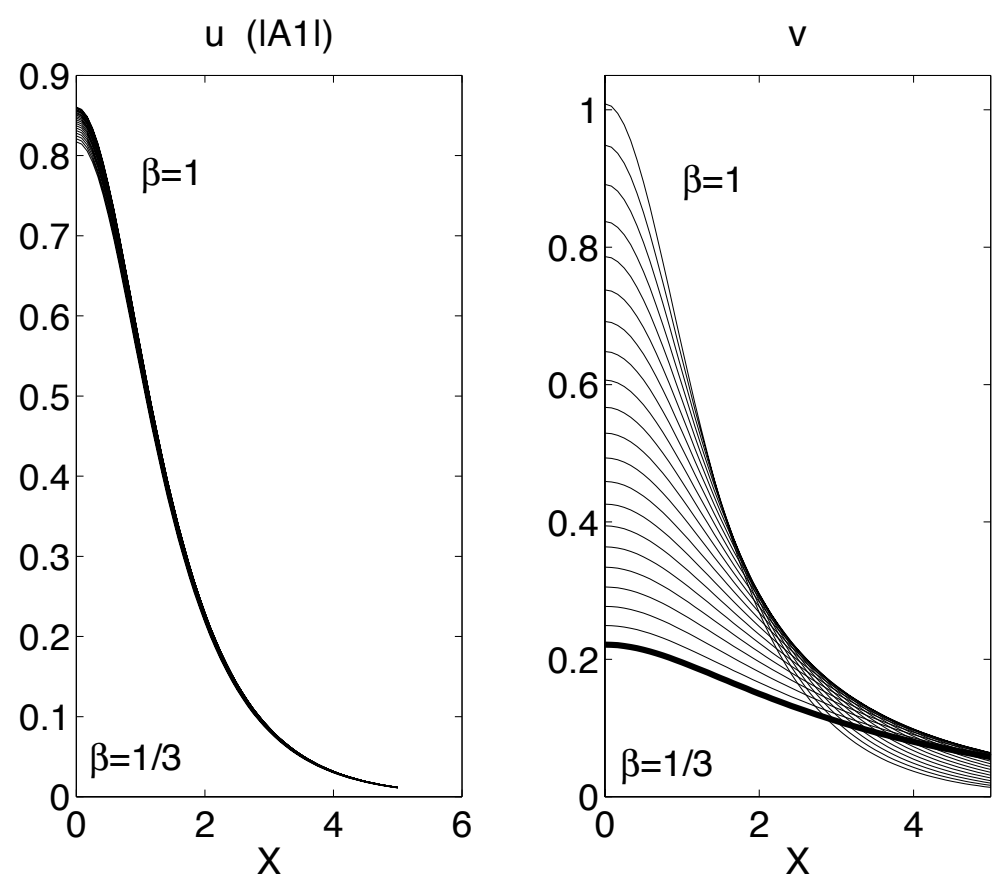

Fig. 1. Plots $u$ (left) and $v$ (right) for the sequence of $\beta$-values, $\beta=\exp \{\log (3)(j-1) / 20\}, j=1,2, \ldots, 21$. Because $u$ changes very little with $\beta$, the 21 graphs for $u$ are almost indistinguishable, visible only a slight thickening of what appears to be a single curve. In contrast, $v$ widens and max $|v|$ decreases with $\beta$. The curve for $\beta=1 / 3$ has been thickened for clarity.

the signs of the cross-modulation terms match those of the self-interaction terms. For geophysical applications, $\alpha_{1}, \alpha_{2}>0$, and this in turn requires that for stable coupled-mode solitons, all the self-interaction coefficients and crossmodulation constants should be positive, too.

Fig. 3 confirms this expectation, at least for equal-frequency solitary waves. Each case was perturbed only by roundoff error. When $\nu_{12}, \nu_{21}>0$, the solitary wave is indeed stable, and the amplitude contours are straight lines paralle to the time axis. When both or even just one of the cross-modulation coefficients is negative, however, the solitary waves fission and separate into two singlemode solitons travelling independently in different modes at different speeds. The negative $v$ has converted the influence of one soliton on that in the other mode into a "potential hill". For a few time units, the coupled-mode solitary wave manages to balance precariously on the top of this, but then the tiny perturbation pushes it away from the top, and the waves roll down the hill (metaphorically) and fission.

When both cross-modulation coefficients are positive, however, the coupledmode solitary wave is stable against even finite amplitude perturbations. Fig. 4 shows the fate of an initial state in which the solitons in different modes were displaced one nondimensional unit apart. The result of their mutual attraction 


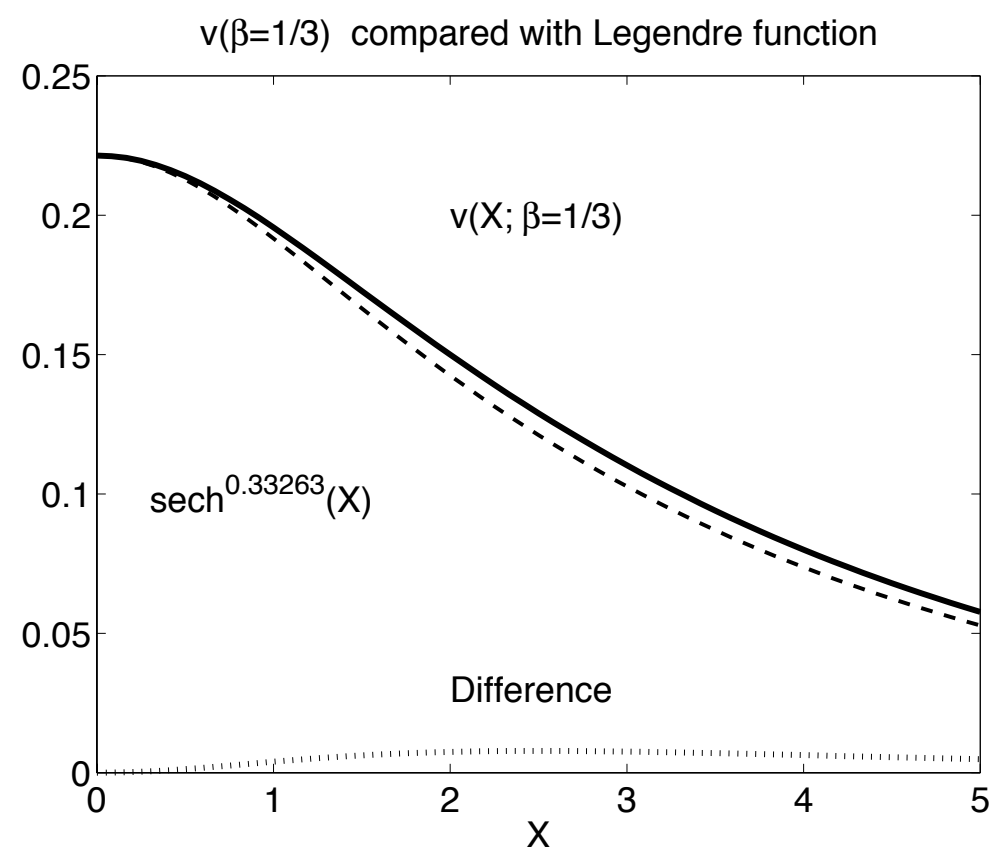

Fig. 2. Thick solid curve: $u(X ; \beta=1 / 3)$. Dashed curve: $v(X ; \beta=0.33263)$, which is an eigenmode of the Associated Legendre equation. The amplitude of the eigenmode has been scaled by $\max |v(X ; 1 / 3)|$ to facilitate the comparison of shapes. Dotted curve: Difference between the solid and dashed curves. The maximum relative error is approximating the solid curve by the Legendre eigenfunction is only $3.5 \%$.

is a stable bound state. However, the offset creates an oscillation, back and forth about the mean position of the peaks in the initial state, which continues indefinitely because the CNLS is non-dissipative. The contour plots at the top are wavy because of the oscillations. We shall these scallop-edged contour line patterns again and again in later figures because it is very common for solitonsoliton collisions to excite one or both of the emergent solitary waves into an oscillating state.

The lower left graph shows the spatial positions of the maxima in each mode as a functions of time, which is the vertical axis. The two modes oscillate in phase so that both the $A_{1}$ and $A_{2}$ are farthest from the spatial origin at the same time. The lower right graph shows as the peaks move from side to side, their amplitudes also fluctuate up and down at the same time, although through a rather small range.

The stability of coupled-mode solitons when both cross-modulation coefficients has been noted previously in the nonlinear optics literature. However, we have been unable to find a rigorous proof. It is possible, for example, that a coupledmode solitary wave might be stable if one cross-modulation were negative but very small and the other was large and positive, the strong attraction of one soliton for the other overcoming the weak repulsion of the second soliton for the first. However, no such examples are known to us. We shall therefore 

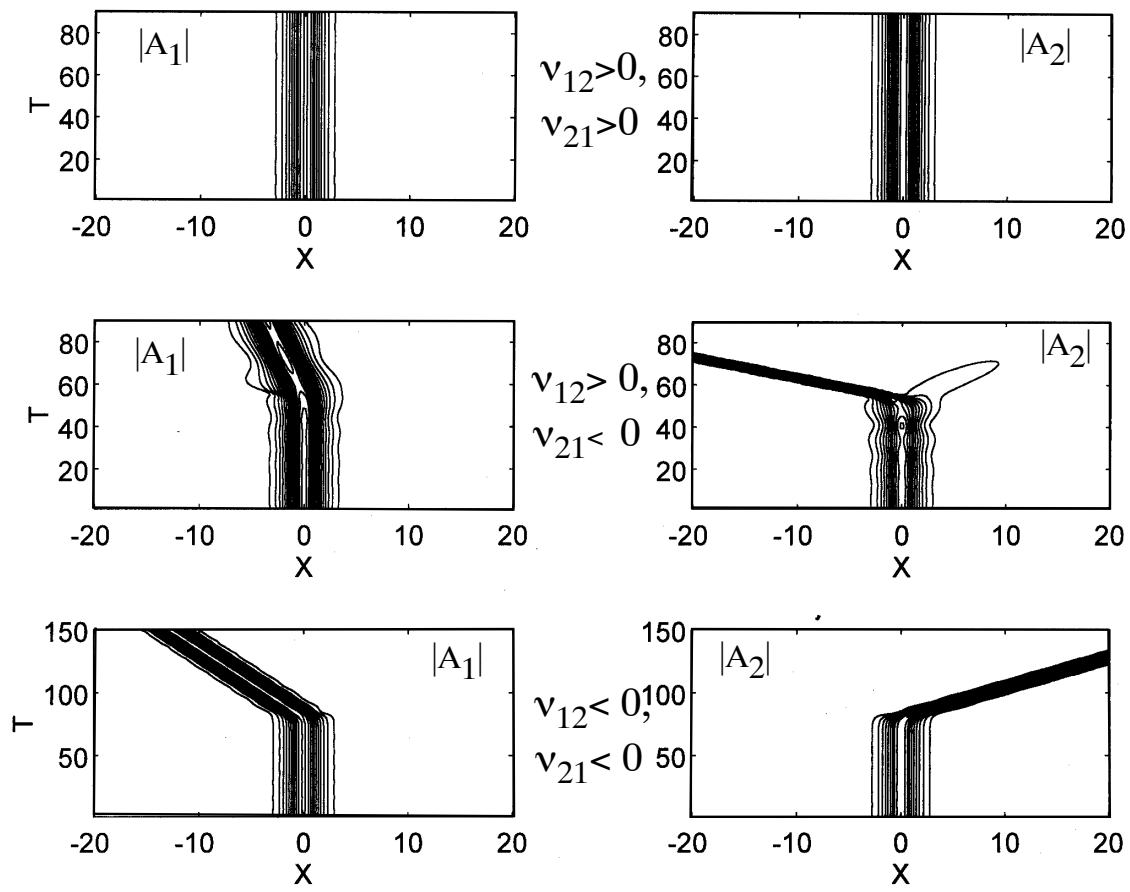

Fig. 3. Contour plots in the $X-T$ plane of amplitude for the coupled-mode envelope solitary wave, perturbed only by roundoff error, for three different sets of signs of the coupling constants: Top: $\nu_{12}, \nu_{21}$ BOTH POSITIVe (STABLE). Middle: $\nu_{12}>0$, $\nu_{21}<0$ ONE POSITIVE, ONE NEGATIVE (FISSIONS). Bottom: $\nu_{12}, \nu_{21}$ BOTH NEGATIVE (FISSIONS). Numerical values of the parameters: (Top) $\alpha_{1}=0.53, \sigma_{1}=1.42$, $\alpha_{2}=0.55, \sigma_{2}=0.93, \nu_{12}=0.11, \nu_{21}=0.23$. (Middle) $\alpha_{1}=0.38, \sigma_{1}=0.38$, $\alpha_{2}=0.55, \sigma_{2}=1.94, \nu_{12}=0.11, \nu_{21}=-0.34$. (Bottom) $\alpha_{1}=0.53, \sigma_{1}=1.42$, $\alpha_{2}=0.51, \sigma_{2}=2.0, \nu_{12}=-0.13, \nu_{21}=-0.23$. The soliton parameters are $V=0$ and $\mu=1$.

study only cases where both cross-modulation coefficients are positive in the remainder of this paper.

\section{$5 \quad$ Numerical Methods}

To study colliding solitary waves, we solved the CNLS system by using a Fourier pseudospectral method cobmined with fourth-order Runge-Kutta time integration as described in $[3,5,12,6]$. We imposed periodic boundary conditions on a spatial domain that was sufficiently large so that the solitary waves were not affected by the boundaries. In most runs, 256 grid points in $X$ were sufficient. 
$\mid \mathrm{A} 1 \mathrm{I}$
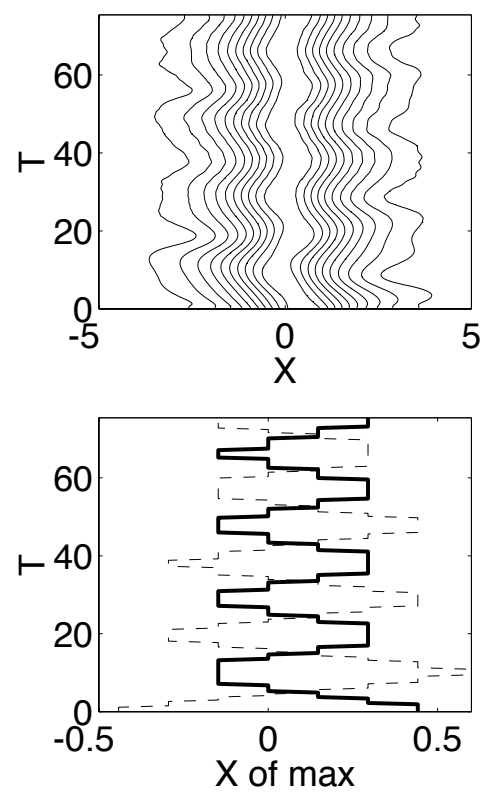

|A2 |
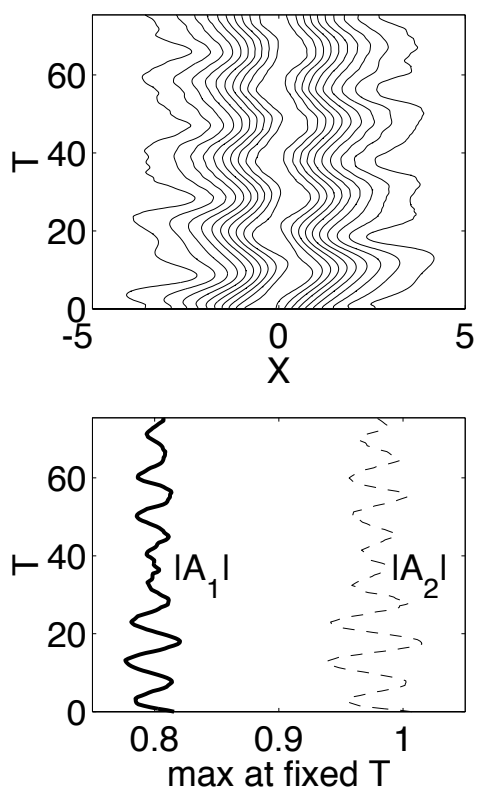

Fig. 4. Stable oscillations of a perturbed coupled-mode soliton. Upper pair of graphs: isolines of $\left|A_{1}(X, T)\right|$ (left) and $\left|A_{2}(X, T)\right|$ (right). The lower left graph shows the positions of the maximum of $\left|A_{1}\right|$ (thick solid curve) and of $\mid A_{2}$ (thin dashed line) at each time. The lower right graph shows the maxima of $\left|A_{1}\right|$ (left) and $\left|A_{2}\right|$ (right) as a function of time (vertical axis). The parameters and initial state are identical to the top pair of graphs in the previous figure except that the positions of the two components were displaced by 1.0 in $X$. The computations were performed on a large spatial interval, $x \in[-12 \pi, 12 \pi]$, but only part of the interval is shown for clarity.

The conservation laws

$$
\frac{d}{d T} \int_{-\infty}^{\infty}\left|A_{1}\right|^{2} d x=0, \quad \frac{d}{d T} \int_{-\infty}^{\infty}\left|A_{2}\right|^{2} d x=0
$$

were used as a numerical check. (Parenthetically, note that Zakharov and Schulman[22] list two additional conservation laws, but these were not needed here.)

To solve the SCNLS system of two ODEs in $X$, we also employed a Fourier pseudospectral algorithm in $X$. This creates a system of nonlinear algebraic equations in which the Fourier coefficients are the unknowns. This system was solved by Newton's iteration. To initialize the iteration, we used two strategies[3-5]. First, the computation of a branch of solutions was begun using one of the analytical approximations described above, such as that for equal-frequency coupled-mode solitons. Second, continuation in a parameter was used to trace the rest of the branch. The parameter was decreased in small steps using the results from previous solutions to initialize the iteration for the current parameter value. Polynomial extrapolation using interpolating 
polynomials of degree up to four, fitted to previously computed solutions, generated excellent first guesses. Until five points on the branch had been computed, we used polynomials of lower degree to extrapolate the initial guess for the next point.

\section{Collisions I: Single-Mode Soliton with Coupled-Mode Solitary Wave}

To perform collision experiments, we need to use the Galilean invariance discussed earlier to write the solitary waves in their most general form, which allows for a non-zero translational velocity $V$. In this section, the initial condition is taken to be the sum of a single-mode soliton, arbitrarily chosen to be in $A_{1}$ plus an equal-frequency coupled-mode solitary wave. The initial condition is

$$
\begin{aligned}
A_{1}(X, 0)= & \mu \sqrt{2 \frac{\alpha_{1} \sigma_{2}-\alpha_{2} \nu_{12}}{\sigma_{1} \sigma_{2}-\nu_{12} \nu_{21}}} \operatorname{sech}(\mu X) \exp \left(i \frac{V}{2 \alpha_{1}} X+i \phi_{1}\right) \\
& +\sqrt{\frac{2 \alpha_{1}}{\sigma_{1}} \eta \operatorname{sech}\left(\eta\left(X+X_{0}\right)\right) \exp \left(i \xi\left(X+X_{0}\right)+i \phi_{3}\right)} \\
A_{2}(X, 0)= & \mu \sqrt{2 \frac{\alpha_{2} \sigma_{1}-\alpha_{1} \nu_{21}}{\sigma_{1} \sigma_{2}-\nu_{12} \nu_{21}}} \operatorname{sech}(\mu X) \exp \left(i \frac{V}{2 \alpha_{2}} X+i \phi_{2}\right)
\end{aligned}
$$

where the initial separation $X_{0}=10$. The absolute velocities of the solitons is not important, although we choose equal and absolute velocities for convenience. The only significant velocity parameter is the relative velocity $\Delta V=2 \alpha_{1} \xi-V$, which is specified in the captions. Since the CNLS solutions are indifferent to multiplication by $\exp (i \Phi)$ for any constant $\Phi$, it follows that we can choose one of the phases arbitrarily without loss of generality; we pick $\phi_{3}=0$.

Unfortunately, we still have too many parameters to explore the parameter space completely. The cases illustrated in Figs. 5-7 are representative of our experiments. We find that the collisions are sensitive to both the initial phase and relative velocity of the interacting solitary waves.

When $\phi_{1}=\phi_{2}=\pi$, Fig 5 shows that the collisions are rather boring at low relative velocity: the two solitons reflect off each other elastically (that is, without loss of energy or change of shape); there are no after-effects except that the relative velocity of each soliton has changed sign (top pair of plots). At medium velocity, the single-mode soliton also elastically reflects, but coupled-mode soliton fissions as its two components in different modes travel at different velocities after the impact. The emerging soliton in $A_{2}$ has been 
greatly broadened and weakened by the collision and displays strong oscillations. At large relative velocity, we have what we have dubbed an "Exchange Reflection". The two incoming solitons in $A_{1}$ reflect elastically, but the part of the coupled-mode soliton in $A_{2}$ does not reflect at all, but instead propagates leftward as part of a new coupled-mode soliton whose $A_{1}$ part was originally the single-mode solitary wave.

When the phases of the coupled-mode soliton are changed to $\phi_{1}=\phi_{2}=\pi / 8$ as in Fig 6, the collisions show some similarities to the previous case: elastic reflection at low velocity, destruction of the coupled-mode soliton at medium velocity, and the propagation of a coupled-mode soliton in the same direction as before the collision at high velocity. However, there are some differences, too. At low velocity (top graphs), the reflected coupled-mode solitary wave is oscillating. At medium velocity, the reflected coupled-mode solitary does not fission. Instead, it becomes highly oscillatory in both modes; the $A_{2}$ part (right

middle plot) continues to radiate away energy indefinitely. At high velocity, we have described the interaction in Table 1 as a "Crossing" rather than an "Exchange" because the interacting solitary waves merge into a single structure during the collision whereas in Fig. 5, the contours of the interacting solitons always remain distinct and spatially separated so that the label "Crossing" seems inappropriate.

In Fig. 7, $\phi_{1}=\phi_{2}=0$. This is the boring case: nearly-elastic crossing at all relative velocities. The coupled-mode soliton oscillates after the collision; rapid oscillations at small relative velocity and slow oscillations after collisions at medium or large velocity. It is remarkable that we can pass from boring to a mix of fission and reflection just by altering the phases of the initial coupled-mode soliton.

\section{Collisions II: Coupled-Mode Solitons}

In this section, we explore the collision of one coupled-mode equal-frequency soliton with another by choosing

$$
\begin{aligned}
A_{1}(X, 0)= & \mu \sqrt{2 \frac{\alpha_{1} \sigma_{2}-\alpha_{2} \nu_{12}}{\sigma_{1} \sigma_{2}-\nu_{12} \nu_{21}}}\left\{\operatorname{sech}(\mu X) \exp \left(i \frac{V_{1}}{2 \alpha_{1}} X\right)\right. \\
& \left.+\operatorname{sech}\left(\mu\left(X+X_{0}\right)\right) \exp \left(i \frac{V_{2}}{2 \alpha_{1}}\left(X+X_{0}\right)+i \phi_{4}\right)\right\} \\
A_{2}(X, 0)= & \mu \sqrt{2 \frac{\alpha_{2} \sigma_{1}-\alpha_{1} \nu_{21}}{\sigma_{1} \sigma_{2}-\nu_{12} \nu_{21}}}\left\{\operatorname{sech}(\mu X) \exp \left(i \frac{V_{1}}{2 \alpha_{2}} X\right)\right. \\
& \left.\operatorname{sech}\left(\mu\left(X+X_{0}\right)\right) \exp \left(i \frac{V_{2}}{2 \alpha_{2}}\left(X+X_{0}\right)+i \phi_{5}\right)\right\}
\end{aligned}
$$


Table 1

Coupled Soliton/Soliton Collisions

Note: "Reflection" means that the soliton velocities change sign. "Elastic" implies that the solitons lose little energy during the collision. "Exchange" means that the Mode 2 component is transferred from its original partner to the other soliton which was initially in Mode 1 only. "Crossing" means that the solitons freely interpenetrate and then continue with unchanged direction.

\begin{tabular}{|c|c|c|c|}
\hline $\begin{array}{l}\text { Relative } \\
\text { Velocity }\end{array}$ & $\begin{array}{c}\phi_{1}=\phi_{2}=\pi \\
\text { Fig. } 5\end{array}$ & $\begin{array}{c}\phi_{1}=\phi_{2}=-\pi / 8 \\
\text { Fig. } 6\end{array}$ & $\begin{array}{c}\phi_{1}=\phi_{2}=0 \\
\text { Fig. } 7\end{array}$ \\
\hline Slow & Reflection (Elastic) & $\begin{array}{c}\text { Reflection (Elastic) \& Promotion } \\
\text { of coupled-mode soliton } \\
\text { to oscillating excited state }\end{array}$ & $\begin{array}{l}\text { Crossing with Promotion } \\
\text { of both solitons to } \\
\text { oscillating excited states }\end{array}$ \\
\hline Medium & $\begin{array}{c}\text { Reflection with Fission } \\
\text { Mode } 2 \text { fragment broadened } \\
\text { and oscillatory }\end{array}$ & $\begin{array}{l}\text { Reflection of much weakened } \\
\text { single-mode soliton; } \\
\text { Coupled-mode soliton travels } \\
\text { much slower after collision; } \\
\text { continuous radiation in mode } 2\end{array}$ & $\begin{array}{l}\text { Crossing (Elastic); } \\
\text { coupled soliton } \\
\text { slowly oscillates }\end{array}$ \\
\hline Fast & $\begin{array}{c}\text { Exchange Reflection } \\
\text { coupled soliton } \\
\text { slowly oscillates }\end{array}$ & $\begin{array}{l}\text { Crossing (Elastic) } \\
\text { coupled soliton } \\
\text { slowly oscillates }\end{array}$ & $\begin{array}{l}\text { Crossing (Elastic) } \\
\text { coupled soliton } \\
\text { slowly oscillates }\end{array}$ \\
\hline
\end{tabular}

where $V_{1}$ and $V_{2}$ are the differing velocities of the two solitary waves generated by these initial conditions. We have arbitrarily specified the phases of the first soliton to be zero and will vary the phases of the second coupled-mode solitary wave.

Fig. 8 shows the collisions when $\phi_{4}=\phi_{5}=0$. At low velocities (top pair of graphs), the solitons form a metastable intermediate: the solitons hit, rebound slightly, and merge again. The metastable state then fissions into three parts: two separate, single-mode solitons in $A_{2}$, which fly away with much larger relative velocities than the initial solitary waves possessed, plus a single, oscillating, non-travelling solitary wave in $A_{1}$. At medium relative velocity, the metastable intermediate is quite long-lived. When it fragments, it dissolves into two coupled-mode solitons with relative velocities a little smaller than the incoming pair. At high velocities, the solitons elastically cross; the emergent solitary waves are unaffected by the collision except for shifts in position and small low-frequency oscillations.

Fig. 9 shows what happens when $\phi_{4}$ is changed to $\pi$ while $\phi_{5}=0$ as before. At low velocities, there is again a very long-lived metastable intermediate 


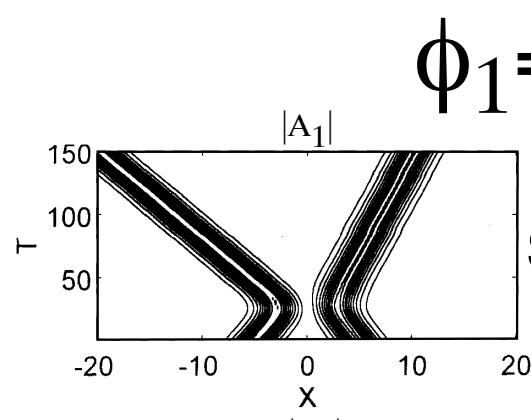

$\phi_{1}=\phi_{2}=\pi$
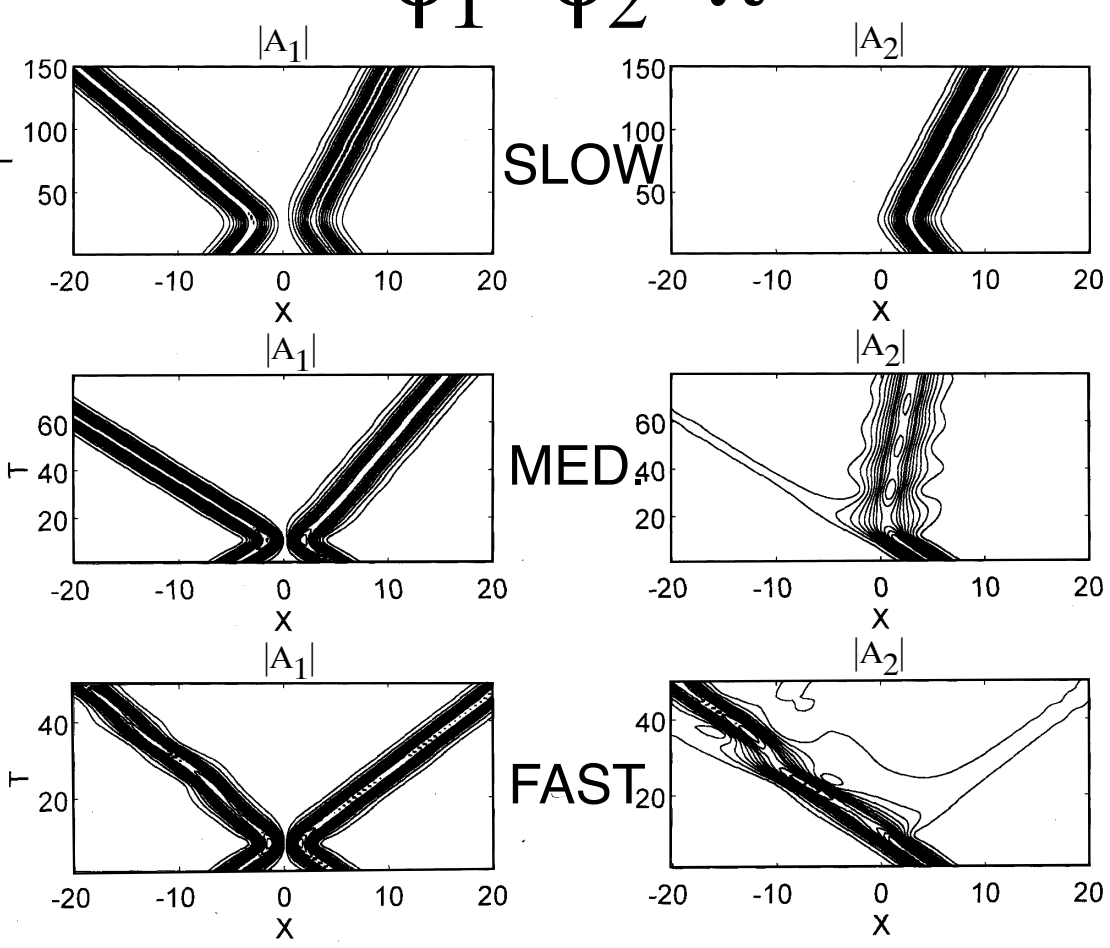

Fig. 5. SINGLE-MODE/COUPLED-MODE COLLISIONS. The single-mode soliton is in $A_{1}$ only, coming from the left. In each pair of contour plots, $\left|A_{1}(X, T)\right|$ is on the left and $\left|A_{2}(X, T)\right|$ on right. The initial relative velocity is 0.2 (top); 0.7 (middle pair), and 1.0 bottom. For all cases in this figure, $\phi_{1}=\phi_{2}=\pi, \phi_{2}=0$ and $X_{0}=10.0$. Coupled-NLS parameters: $\alpha_{1}=0.53, \sigma_{1}=1.42, \alpha_{2}=0.55, \sigma_{2}=0.93$, $\nu_{12}=0.11$ and $\nu_{21}=0.23 . \mu=\eta=1$.

state which does not propagate, but does show several oscillations before it splits into two coupled-mode solitons. These are noticeably oscillatory and have much higher relative velocities away from each other than the incoming solitons had towards each other. At higher relative velocity, the metastable intermediate disappears; indeed, the contours of $\left|A_{1}\right|$ never merge. The solitons reflect almost elastically from one another although the outgoing solitons do shown small-amplitude oscillations. No plot is shown for high relative velocity because it is very similar to that for intermediate $\Delta V$.

Fig. 10 is different from previous contour plots in that the relative velocity is kept fixed while three different sets of phases of the second coupled-mode soliton are applied. All graphs are qualitatively similar in the sense that the solitons merely reflect off each other: the collisions are nearly elastic and the both solitary waves emerge almost unscathed except for the change of direction.

However, there are some interesting differences. One might suppose that the 


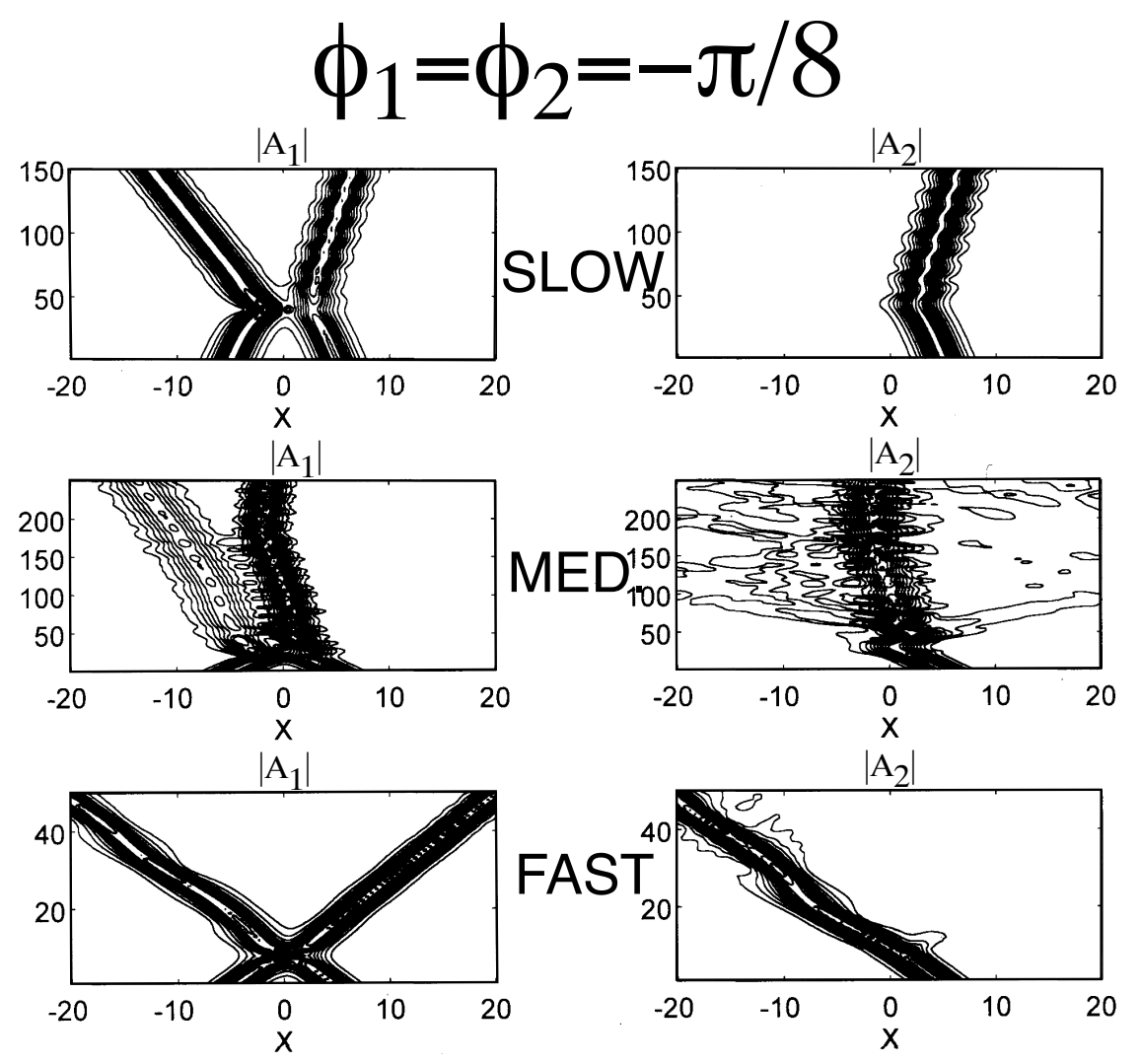

Fig. 6. Same as Fig. 5 except that $\phi_{1}=\phi_{2}=-\pi / 8$. The relative velocity varies from 0.1 (top) to 0.3 (middle) to 1.0 (bottom).

degree of overlap or interpenetration of the solitary waves at that time of closest approach would controlled primarily by relative velocity: at high velocity, the solitons would completely merge while in slow collisions, the solitons might reflect without ever strongly overlapping. Fig. 10 shows that this conjecture is false: the relative velocities are the same but the degree of overlap varies strongly between the three cases, all because of the variations in the phase of the second solitary wave. In the top graph, none of the contours of the solitary waves overlap at all. In the middle and lower graphs, however, the solitons temporarily form a single structure which fissions immediately into the reflected solitons.

The emerging solitary waves in the top case $\left(\phi_{4}=\phi_{5}=\pi\right)$ show no visible oscillations. However, the emerging solitary waves in the other two graphs display visible internal oscillations - very strong for the bottom case. The latter pair of solitons also emerge with significantly larger relative velocities than the two incident solitary waves whereas the other two graphs show solitons whose velocities have been little altered, except for sign, by the collision. 

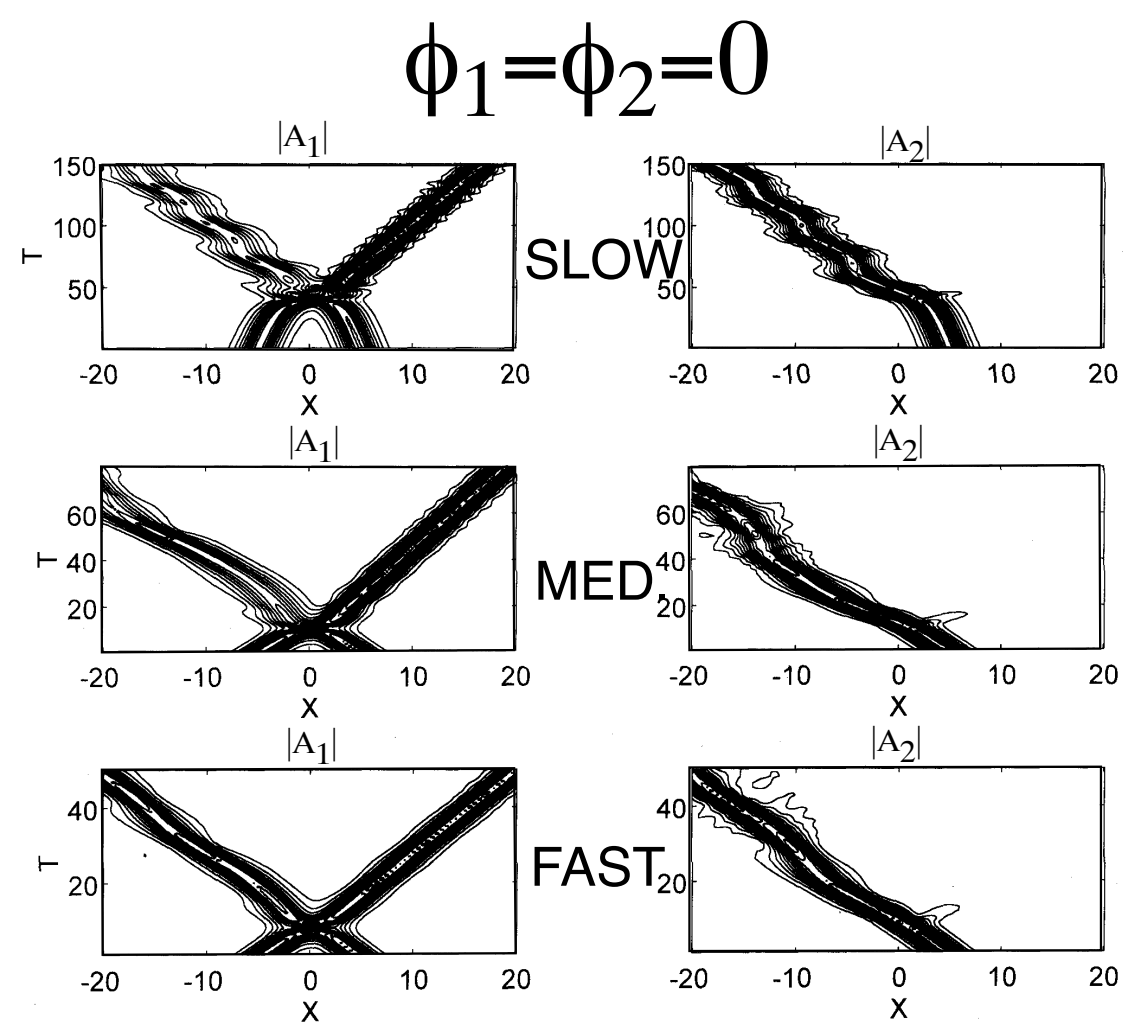

Fig. 7. Same as Fig. 5 except that $\phi_{1}=\phi_{2}=0$.

\section{Concluding Remarks}

Through cross-modulation, envelope wave packets in different modes, such as Rossby waves in different latitudinal or vertical modes of the atmosphere, can be coupled into coherent structures. We numerically show that when but only when - both cross-modulation coefficients are positive, the coupledmode solitary waves are stable to perturbations. When one or both coefficients are negative (relative to the signs of the corresponding dispersion coefficients $\alpha_{j}$ ), the coupled-mode solitary wave is unstable to spontaneous fission into two independent solitary waves, one in each mode, of different velocities.

When the coupled-mode soliton collides with either a single-mode solitary wave or another coupled-mode soliton, the result is sensitive to both the initial phases and relative velocities of the colliding wave packets. Elastic reflection, fission-after-collision, exchange of components between the single-mode and coupled-mode solitary wave, death-by-slow-radiation, and strong post-collision oscillations are all observed.

In spite of the experiments given here and a large body of work in nonlinear optics, the full parameter space of the Coupled-NLS system is still unexplored. 
Table 2

Coupled Soliton/Coupled Soliton Collisions

Note: "Reflection" means that the soliton velocities change sign. "Elastic" implies that the solitons lose little energy during the collision. "Exchange" means that the Mode 2 component is transferred from its original partner to the other soliton which was initially in Mode 1 only. "Crossing" means that the solitons freely interpenetrate and then continue with unchanged direction.

\begin{tabular}{|c|c|c|}
\hline $\begin{array}{l}\text { Relative } \\
\text { Velocity }\end{array}$ & $\begin{array}{c}\phi_{4}=\phi_{5}=0 \\
\text { Fig. } 8\end{array}$ & $\begin{array}{c}\phi_{4}=\pi, \phi_{5}=0 \\
\text { Fig. } 9\end{array}$ \\
\hline Slow & $\begin{array}{l}\text { Metastable Intermediate; } \\
\text { Fission into } 3 \text { Fragments }\end{array}$ & $\begin{array}{c}\text { Reflection with } \\
\text { Metastable Intermediate } \\
\text { Big Velocity Change in Both } \\
\text { Solitons }\end{array}$ \\
\hline Medium & $\begin{array}{l}\text { Metastable Intermediate; } \\
\text { Splits into two } \\
\text { Coupled-Mode Solitons }\end{array}$ & $\begin{array}{c}\text { Reflection; } \\
\text { Promotion of Both Solitons } \\
\text { into Oscillating States }\end{array}$ \\
\hline Fast & Crossing & $\begin{array}{c}\text { Reflection; } \\
\text { Promotion of Both Solitons } \\
\text { into Oscillating States }\end{array}$ \\
\hline
\end{tabular}

This work was supported by the National Natural Science Foundation of China through grant 49375239 and by the American National Science Foundation through grant OCE9521133.

\section{References}

[1] G. P. Agraval, Nonlinear Fiber Optics, Academic, Boston, second ed., 1995.

[2] N. Akhmediev And A. Ankiewicz, Novel soliton states and bifurcation phenomena in nonlinear fiber couplers, Phys. Rev. Lett., 70 (1993), pp. 23952398.

[3] J. P. Boyd, Chebyshev and Fourier Spectral Methods, Springer-Verlag, New York, 1989. $792 \mathrm{pp}$.

[4] - Weakly Nonlocal Solitary Waves and Beyond-All-Orders Asymptotics: Generalized Solitons and Hyperasymptotic Perturbation Theory, vol. 442 of Mathematics and Its Applications, Kluwer, Amsterdam, 1998. 608 pp.

[5] — Chebyshev and Fourier Spectral Methods, Dover, New York, 1999. Second edition of Boyd(1989a), to appear. 


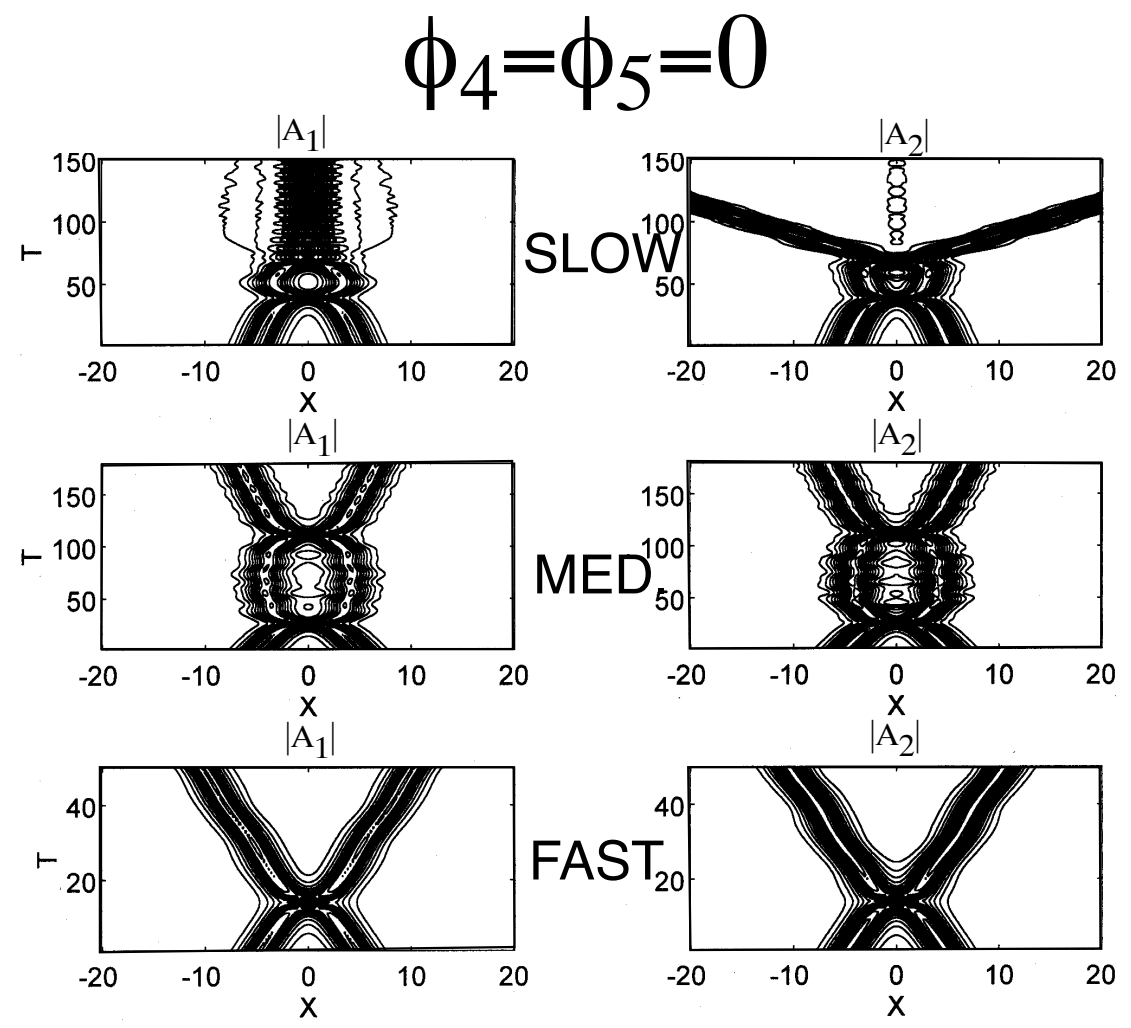

Fig. 8. COUPLED-MODE/COUPLED-MODE COLLISIONS. Contour plots of $\left|A_{1}\right|$ (left graphs) and $\left|A_{2}\right|$ (right) in the $X-T$ plane. The phase parameters of both solitons are zero. Relative initial velocity: (Top) 0.1, (Middle) 0.2 and (Bottom) 0.5.

[6] B. Fornberg, A Practical Guide to Pseudospectral Methods, Cambridge University Press, New York, 1996.

[7] M. Haelterman And A. P. Sheppard, Bifurcation phenomena and multiple soliton-bound states in isotropic Kerr media, Phys. Rev. E, 49 (1994), pp. 33763381.

[8] A. Hasegawa And Y. Kodama, Solitons in Optical Communication, Clarendon Press, Oxford, 1995.

[9] L. A. Lugiato, Transverse nonlinear optics - introduction and review, Chaos, Solitons and Fractals, 4 (1994), pp. 1251-1258.

[10] S. V. Manakov, On the theory of two-dimensional stationary self-focusing of electromagnetic waves, Soviet Physics JETP, 38 (1973), pp. 248-253.

[11] C. R. Menyuk, Stability of solitons in birefringent optical fibers. II. Arbitrary amplitudes, J. Opt. Soc. Am. B, 5 (1988), pp. 392-402.

[12] V. I. Pulov, I. M. Uzunov, and E. J. Chacarov, Solutions and laws of conservation for coupled nonlinear Schrödinger equations: Lie group analysis, Phys. Rev. E, 57 (1998), pp. 3468-3477. 


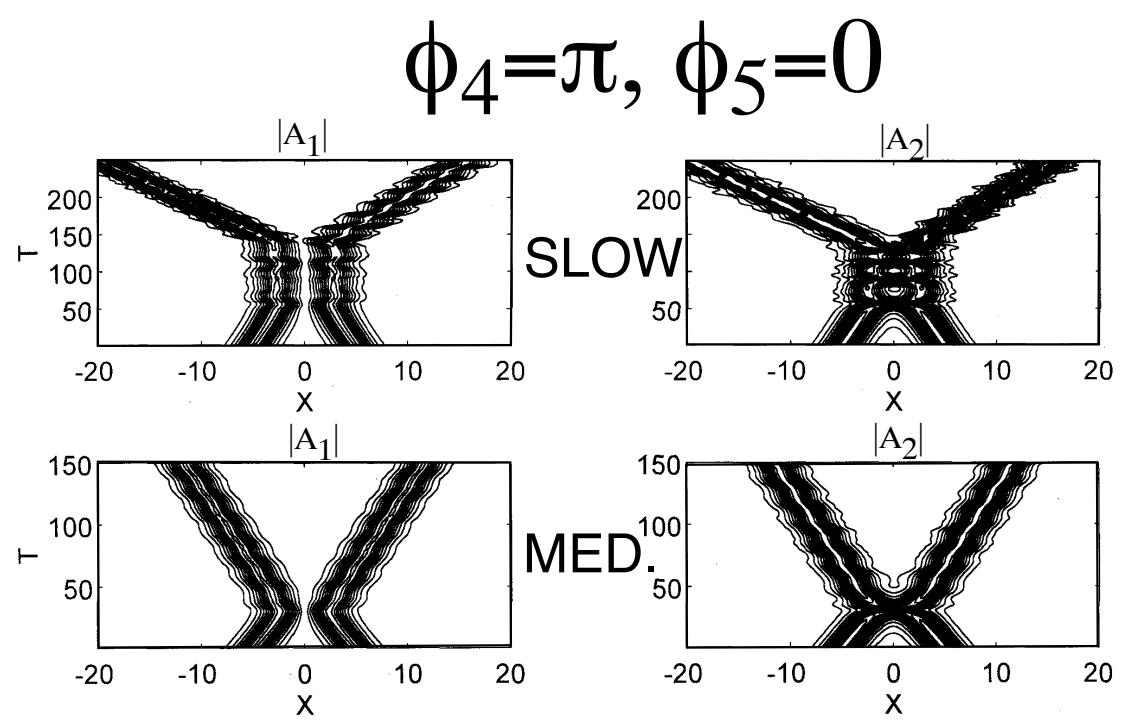

Fig. 9. As in Fig. 8 except that $\phi_{4}=0, \phi_{5}=0$. Top: Initial velocity 0.1. Bottom: relative velocity 0.2 . No graph for high velocity is shown because it is very similar to the bottom pair of plots.

[13] R. Radhakrishnan, R. Sahadevan, and M. Lakshmanan, Integrability and singularity structure of coupled nonlinear Schrodinger equations, Chaos, Solitons and Fractals, 5 (1995), pp. 2315-2327.

[14] B. F. Sanders, N. Katopodes, and J. P. Boyd, Unified pseudo-spectral solution to water wave equations, Journal of Hydraulic Eng. ASCE, 124 (1998), pp. $2-12$.

[15] B. TAN, Collision interactions of envelope Rossby solitons in a barotropic atmosphere, J. Atmos. Sci., 53 (1996), pp. 1604-1616.

[16] B. TAN AND S. LIU, Collision interactions of solitons in a baroclinic atmosphere, J. Atmos. Sci., 52 (1995), pp. 1501-1512.

[17] M. V. Tratnik and J. E. Sipe, Bound solitary waves in a birefringent optical fiber, Phys. Rev. A, 38 (1988), pp. 2011-2017.

[18] I. M. Uzunov, R. Muschall, M. Gölles, Y. S. Kivshar, B. A. Malomed, AND F. Lederer, Pulse witching in nonlinear fiber directionalcouplers, Phys. Rev. E, 51 (1995), pp. 2527-2537.

[19] J. YAnG, Coherent structures in weakly birefringent nonlinear optical fibers, Stud. Appl. Math., 97 (1996), pp. 127-148.

[20] — Vector solitons and their internal oscillations in birefringent nonlinear optical fibers, Stud. Appl. Math., 98 (1996), pp. 61-97. 

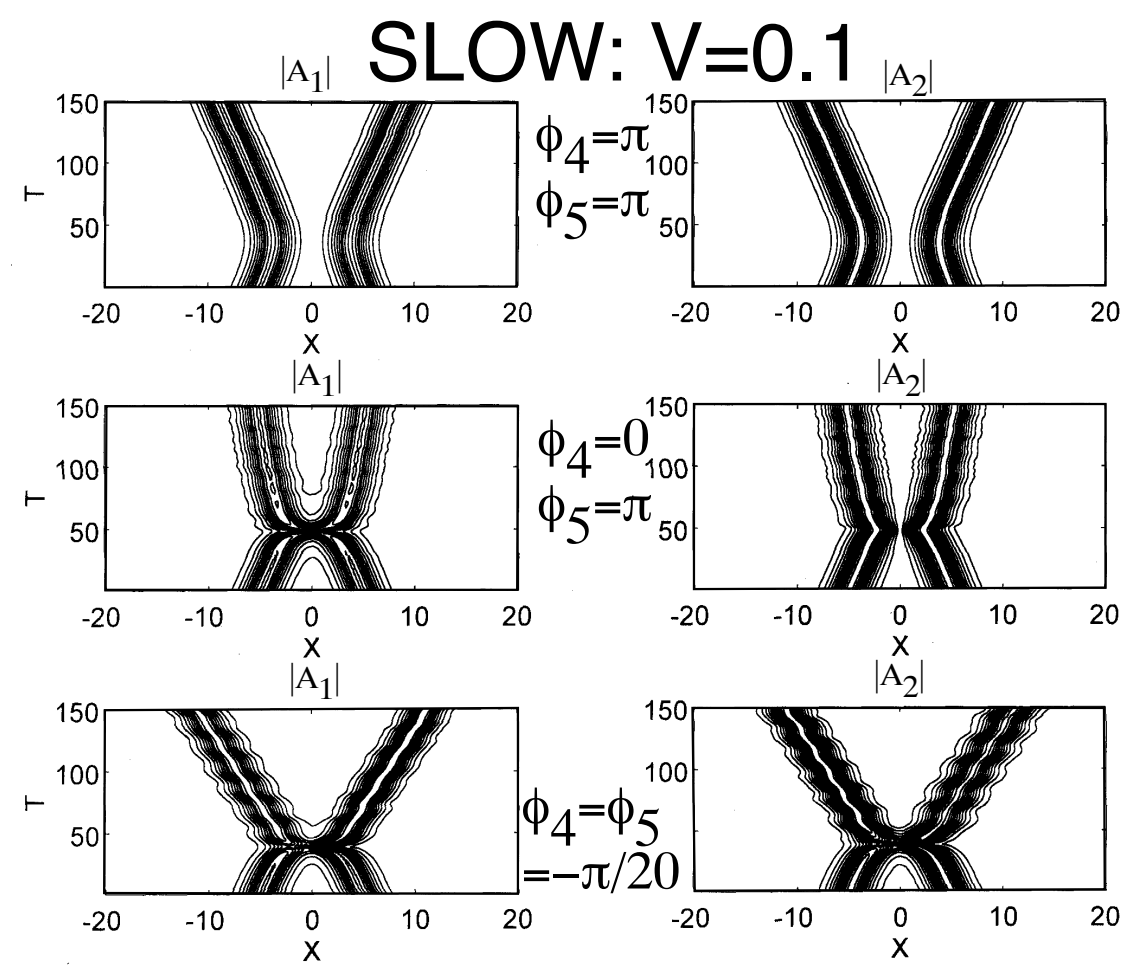

Fig. 10. As in Fig. 8 except that the VELOCITY is fixed (at $V=0.1$ ) and the PHASES of the second coupled-mode soliton are varied. Top: $\phi_{4}=\phi_{5}=\pi$. Middle: $\phi_{4}=0, \phi_{5}=\pi$. Bottom: $\phi_{4}=\phi_{5}=-\pi / 20$.

[21] — Classification of the solitary waves in coupled nonlinear Schrödinger equations, Physica D, 108 (1997), pp. 92-112.

$[22] \_$, Multiple permanent-wave trains in nonlinear systems, Stud. Appl. Math., 100 (1998), pp. 127-152.

[23] J. YAng And D. J. Benney, Some properties of nonlinear wave systems, Stud. Appl. Math., 96 (1996), pp. 111-139.

[24] V. E. Zakharov and E. I. Schulman, To the integrability of the system two coupled nonlinear Schrödinger equations, Physica D, 4 (1982), pp. 270-274. 